\title{
CRÍTICA DE LA AUTORIDAD FACTUAL. POR LA ADMINISTRACIÓN DE LOS HOMBRES CONTRA LA ADMINISTRACIÓN POR LAS COSAS
}

\author{
Danilo Martuccelli ${ }^{1}$
}

\section{Resumen / Abstract}

El artículo propone un análisis de lo que caracteriza como una de las grandes tendencias contemporáneas en el ejercicio de la autoridad: el tránsito de las formas relacionales de la autoridad en dirección de la autoridadfactual. Tras una caracterización de esta última, tres grandes mecanismos son estudiados: el tracking, o el gobierno de las conductas a través de las trazas pasadas de un actor; los dispositivos, en tanto que mecanismos que controlan a los individuos haciéndoles hacer cosas bajo la forma de meras exigencias de funcionalidad; las ligaduras, como procesos que restringen y subordinan las decisiones futuras al respeto de ciertos indicadores. Por último, el artículo señala las especificidades de los retos que la expansión de este tipo de autoridad engendra para la crítica social.

Palabras clave: autoridad, tracking, dispositivos, ligaduras, factual.

\section{CRITIQUE OF FACTUAL AUTHORITY. FOR THE ADMINISTRATION OF MEN AGAINST THE ADMINISTRATION FOR THINGS}

Thispaper analyzes what proposes to be a major contemporary trend in the exercise of authority: the transit from relational authority to factual-authority. After a characterization of the latter, three of its main mechanisms are studied: tracking, or the government of individuals behavior through the past traces leaved by an actor; apparatus, mechanisms controlling individuals making them do things responding to mere functional requirements; ties, processes that constrain and subordinate future decisions to certain indicators. Finally, the

1 Université Paris Descartes, IUF, CERLIS-CNRS. E-mail : danilo.martuccelli@parisdescartes.fr 
paper points out the specific challenges that the expansion of this type of authority breeds for social criticism.

Keywords: authority, tracking, apparatus, bonds, factual.

\section{La autoridad ¿más allá de Marx y Weber?}

El tema de la autoridad, como la noción cercana de dominación, continúa inscribiéndose, incluso de manera crítica, en la descendencia de los trabajos de Marx y de Weber. Del primero -Marx- se retiene sobre todo que la noción designa un tipo particular de relación social marcada por una forma de subordinación que, en la modernidad, no es solamente de índole personal (como fue la relación entre el amo y el esclavo), sino que toma más bien la forma de una subordinación impersonal a restricciones sistémicas -como las del tipo capital-trabajo. Un proceso asegurado conjuntamente por coerciones y por diversos mecanismos de extorsión del consentimiento de los subordinados (por medio de la ideología dominante) al orden de las cosas. Estos dos elementos - coerción y consentimiento- delimitan la estructura básica de la dominación, y por esta vía, dan cuenta -incluso de manera muchas veces sólo implícita- de los procesos de autoridad.

La producción del consentimiento a través del trabajo ideológico de inculcación es sin duda un aspecto central del pensamiento marxista. Sin embargo, es probable que el principal mérito de Marx en este punto haya sido el haber comprendido la transformación inducida por la aparición de formas de subordinación impersonal por un lado, y tras ellas, y por el otro lado, por el tránsito de una mera subordinación "formal" del trabajo (propia, por ejemplo, a las manufacturas, sancionada por la jornada del trabajo pero sin existencia efectiva en los talleres), a una subordinación "real", propia de la industria moderna, basada en un conjunto constante de controles durante todo el proceso de producción (Marx, 1977). En Marx, a término, la dominación-autoridad reposa sobre una serie de procesos estructurales que limitan del "exterior" - gracias a las coerciones justamente- las acciones de los subordinados. Sin embargo, y a pesar de lo anterior, en el fondo, durante mucho tiempo se supuso que la mantención de la dominación-autoridad -cualquiera que sea la fuerza 
de las coerciones- necesitaba de la adhesión cultural de los subordinados, es decir una forma de servidumbre o sumisión voluntaria.

El segundo -Weber (1971) - ha trazado el corazón de la problemática de la autoridad desde hace un siglo: la autoridad reposa siempre sobre el reconocimiento, por los individuos gobernados, de lo bien fundado de un ejercicio de poder sobre ellos. Este reconocimiento puede ser inconsciente, tácito, crítico, explícito, reflexivo, pero es su presencia lo que permite en el fondo hablar de autoridad. La autoridad es pues lo que hace que el poder de unos sobre otros se vuelva legítimo. La gran fuerza de Weber fue comprender con toda la profundidad necesaria el cambio introducido por la revolución democrática. En el orden tradicional y en sociedades jerárquicas, la autoridad es una evidencia cotidiana garantizada por el peso de la tradición, el valor de los ancestros y en última instancia por un garante meta-social, religioso y ultra-mundano. Por el contrario, en una sociedad sumida, como lo afirma Weber, en el desencantamiento, la autoridad se queda sin fundamento: ¿cómo producir entonces la autoridad en sociedades cada vez más basadas en el ideal de la igualdad? La radicalidad de esta mirada explica por qué, después de un siglo y de innumerables trabajos, la cuestión sociológica de la autoridad no se haya modificado en sus bases conceptuales. El problema cardinal sigue siendo el mismo: ¿cómo hacer, una vez que Dios ha muerto, para que todo no sea permitido? ¿Cómo producir la autoridad en medio de sociedades cada vez menos jerárquicas y cada vez más basadas en la igualdad? Como se sabe, la respuesta weberiana, una vez distinguida tres grandes formas de autoridad (tradicional, carismática y racional-legal) es afirmar la existencia inequívoca de una tendencia central en los tiempos modernos hacia el primado de una de ellas, la autoridad racional-legal -ella misma, es importante subrayarlo, una mezcla de procedimientos jurídicos y legales por un lado, y de consideraciones técnico-científicas por el otro.

A pesar de diferencias notorias e inequívocas, algo es en el fondo común, en las reflexiones sobre la autoridad tanto de Marx como de Weber. En ambos la autoridad está vinculada con la dominación, y por momentos, incluso, la frontera es problemática entre ambas nociones. Cierto, al respecto el principal mérito de la concepción weberiana (sobre la posición de Marx), es que propone una noción de autoridad que, no sin ambigüedad, llama la atención sobre el hecho que es imposible por un lado reducir la autoridad a la dominación y por 
el otro, disociar enteramente una de otra. Si no se conserva esta frontera, se cae, inevitablemente en lecturas doblemente esquemáticas y simplistas.

Por un lado, si se diluye esta frontera, la autoridad se convierte en una mera máscara de la dominación, una tentación presente en los trabajos de Marx, en donde la autoridad se diluye por momentos en la mera disimulación ideológica gracias a la cual se obtiene el consentimiento de los subordinados. En su versión más extrema, la autoridad, en el sentido preciso del término, no existe. Sólo hay formas de dominación y lo esencial es desenmascarar los procesos por los cuales se perpetúa la servidumbre voluntaria. Bien vistas las cosas, lo que se elimina de cuajo es la problemática misma de la autoridad -el hecho que individuos jurídicamente iguales puedan racional y libremente consentir u obedecer ciertas reglas. Si se obvia esta dimensión toda la especificidad del derecho del trabajo moderno, por ejemplo, desaparece analíticamente: a saber, y por ambiguo que sea, la voluntad de enmarcar jurídicamente, dentro de las relaciones asimétricas, el hecho que un individuo ponga a disposición de otro una parte de su tiempo (y dentro de ese lapso temporal su consentimiento implícito a un cierto número de tareas). Pero el otro lado, si se separan radicalmente ambas realidades, o sea, si la autoridad se presenta desprovista de todo residuo en términos de dominación (como es muchas veces el caso en versiones liberales, incluso republicanas y por supuesto funcionalistas), la autoridad se vuelve el mero "aceite" necesario para el buen funcionamiento de la sociedad.

Haremos la hipótesis que para comprender la autoridad en el mundo contemporáneo es preciso situarse en la estela de las reflexiones que venimos, brevemente, de evocar: (1) reconocer la importancia decisiva de las coerciones reales e impersonales en el ejercicio de la autoridad; (2) delimitar la autoridad en torno a un consentimiento explícito cualquiera que sea su procedimiento; (3) preservar la necesaria tensión, por ambigua que sea, entre la autoridad y la dominación. Es en la plena vigencia y continuidad de estos principios como es preciso dar cuenta de la profundidad de la transformación en curso.

En efecto, en muchos ámbitos de la vida social, a lo que estamos asistiendo es a un cuestionamiento de la manera habitual como las ciencias sociales pensaron la autoridad, propiciando la dilución masiva de la problemática de la autoridad detrás de la mera funcionalidad de las cosas. En este marco, se estipula no solamente una separación radical entre la dominación y la autoridad, sino que la temática misma del reconocimiento -tema fundamental de la problemática de la autoridad- tiende a desaparecer en beneficio de lo que 
se presenta como una mera aceptación por los actores, a causa de exigencias funcionales y pragmáticas, de lo que "son las cosas". La subordinación se difumina detrás de una mera aceptación (ya no por el peso de las tradiciones), sino por la fuerza misma de las cosas -en su objetividad intrínseca si se puede decir, en su carácter puramente factual. Vale decir: en la realidad misma de las cosas y de cómo son las cosas ${ }^{2}$.

Cierto, premisas de este proceso pueden advertirse en el análisis que Weber da de la autoridad racional-legal o Marx de la reificación capitalista, sin embargo, un cambio fundamental se ha producido. Para comprenderlo, es preciso apoyarse una vez más en una notable coincidencia entre Marx y Weber: en ambos la autoridad es siempre una relación social entre individuos, por impersonal que sea la subordinación ${ }^{3}$. De ahí, justamente, la centralidad del tema de la ideología en uno, y de la legitimidad en el otro; pero también el esfuerzo de cada uno de ellos en describir, o desvelar, las coerciones reales -y relacionales- por las cuales se ejerce la autoridad. Es aquí donde se inscribe una de las grandes novedades del período contemporáneo. La problematización de la autoridad-relacional, sin obviamente desaparecer, tiende a ser complementada -y en algunos casos, a ser remplazada- por la extensión sin precedentes de la autoridad-factual. Detrás de este proceso, como lo veremos, a lo que se asiste es a un debilitamiento de los grandes postulados de la autoridad como consentimiento (ideología o legitimidad) en beneficio de un ejercicio de la autoridad que se pretende únicamente basado en aspectos factuales y técnicos. Más simple: la autoridad dejaría de ser un asunto normativo y relacional para volverse un tema meramente fáctico.

\section{Las tres formas de la autoridad-factual}

La generalización de las prácticas de autoridad-factual debe comprenderse en el marco del advenimiento más general de una sociedad de

2 En verdad, la autoridad-factual debe entenderse en relación con la metamorfosis observable a nivel de la dominación: a saber, el tránsito de los mecanismos que aseguraron por consentimiento la reproducción del orden social (ideología, habitus, hegemonía, legitimidad...) en beneficio de la primacía de los factores propiamente coercitivos (Martuccelli, 2001 y 2007). Sobre esta base, pero yendo más allá de esta realidad, la autoridad-factual propone una nueva gestión del mando y de la obediencia.

3 Evitemos todo malentendido: en un registro utópico Marx afirma en la Critica al Programa de Gotha (1875) que desaparecidas las clases, el Estado propiamente dicho desaparecería dando paso a instituciones puramente administrativas. "El gobierno de los hombres es sustituido por la administración de las cosas". Pero lo esencial de sus escritos se inserta en torno al problema de la dominación y de la autoridad-relacional entre los hombres. 
la información y del conocimiento, y del papel creciente del conocimiento en la gestión de la sociedad. Pero dentro de este proceso general, un conjunto de estudios y de prácticas subrayan una tendencia particular: la integración y el funcionamiento de la sociedad dejarían de operar por autoridades y acuerdos deliberativos o normativos, y serían cada vez más un mero asunto de ingeniería factual ${ }^{4}$. Tres grandes formas de la autoridad-factual pueden ser distinguidas (tracking, dispositivos, ligaduras): claramente distintas entre sí, e involucrando diferentes temporalidades en el control de las conductas, todas ellas van empero hacia una misma dirección -la eclosión del espacio de la crítica ordinaria a la cual está sometida la autoridad relacional.

\section{Tracking}

El primer tipo de autoridad-factual diluye -en verdad transforma- el espinoso tema de la obediencia de los subordinados en un mero asunto de seguimiento temporal de trazas. El principio es simple: es a través de las trazas exteriorizadas por un actor, y su análisis en tiempo real y secuencial, como es posible prever sus acciones futuras o sus gustos personales. El análisis del historial de trazas plurales dejadas en el mundo por un actor permitiría una forma de conocimiento inédito de los sujetos y sus acciones (de su movilidad, de su consumo, de su consumo energético, de sus estados fisiológicos o psicológicos, etc.). Las técnicas del tracking se encuentran así en la base de lo que se denomina el 2uantified Self (Gadenne, 2012). Demos un ejemplo muy simple: el análisis detallado del ticket de compra en un supermercado permite descubrir muchos rasgos del usuario -edad, sexo, estado de salud (dietas particulares), religión (si compra o no productos cacher o halal), talla de la familia, vegetariano o no, si tiene o no animales domésticos, estilo de vida inducido por las horas en las que hace su compra, y, por supuesto, nivel de ingresos. El ejemplo es voluntariamente simple en este punto de la argumentación, pero permite comprender lo que subyace a estas técnicas: el cruce exponencial de trazas diversas permitiría un conocimiento, exhaustivo y predictivo, de un nuevo tipo, de los individuos. Se trata nada más y nada menos

4 Una tensión de este tipo está presente, por supuesto, en la obra de Habermas (1987) y en su tesis de la colonización de la integración social y del mundo de la vida por los sistemas funcionales y la integración sistémica. A pesar de la justeza de esta tradición, la formulación habermasiana corre empero el riesgo, como lo veremos, de desconocer la verdadera novedad del proceso contemporáneo en curso, y la importancia que para entenderlo debe otorgársele a los "objetos" -a los elementos meramente factuales- por los que se pone en práctica. 
que del advenimiento de una nueva concepción del Yo y de la inteligencia de sus acciones.

Un mecanismo de comprensión que sólo se ha vuelto posible en las últimas décadas, gracias a la expansión de poderosos algoritmos capaces de analizar información en cantidades inmensamente superiores y a una velocidad insospechada a las posibles en un pasado incluso muy próximo.

Para algunos, este proceso se hallaría en el corazón de la revolución que los Big Data introducen en la vida social (Mayern Schönberger, Cukier, 2013). En lo que aquí nos concierne, lo importante es comprender la revolución que esto implica a nivel del control de los actores, y tras ello, de la temática de la autoridad. Para entenderlo es imperioso aprehender la profundidad del cambio epistemológico en curso. Los Big Data, y tras él, nociones como las de 2uantified Self, se inscriben en la radicalización del cuestionamiento de la relación causa-efecto como pivote central de la explicación de los fenómenos, en beneficio de una concepción "pragmática" del conocimiento (lo importante es "lo que marcha"), que tiende a prever las futuras conductas de un individuo a partir de las trazas de sus acciones pasadas, apoyándose en la enorme capacidad, vuelta técnicamente posible por los Big Data, que permiten cruzar un número impresionante de datos (trazas del Yo), muchas veces, sin ninguna relación "intuitiva" entre ellos. A diferencia de la ciencia "clásica", el conocimiento se desliga de la búsqueda de relaciones causales (incluso complejas y no lineales) y se construye alrededor de un cruce exponencial de correlaciones.

Entendámoslo bien. En estadística se sabe que una correlación no es una causa: una vez que una correlación se observa entre dos fenómenos es imperioso descubrir si existe -o no- un vínculo "causal" entre ellos. En el mundo del tracking (y su lógica de correlaciones universales) esta exigencia simplemente desaparece. Se sabe así, por ejemplo, que los algoritmos cruzan factores que no tienen "en apariencia" nada que ver, pero se espera que dada la masa de datos cruzados surjan, por razones que son y permanecen opacas, capacidades reales de predicción a nivel de las conductas futuras.

Se trata de una verdadera revolución epistemológica que es importante diferenciar de la antigua hermenéutica de los signos. En ella, sobre todo en la Edad media -piénsese en la teología negativa-, o más tarde en la tesis de Galileo para quien el libro de la naturaleza estaba escrito en caracteres matemáticas, era aún activa la idea que los signos daban cuenta de la verdadera razón del mundo. A lo que se entra con el tracking es a un universo diferente, uno en el 
cual las vertiginosas capacidades de cálculo substituyen al conocimiento -un universo de pura jungla de trazas que no hacen más "signo" en dirección de una "razón".

¿Por qué la generalización de esta técnica transforma el ejercicio de la autoridad? Porque da pie a la tesis de un gobierno de los hombres que podría hacerse independientemente de sus conciencias, gracias a la mera administración por las cosas. Ya no sería necesario conocer ni las normas que moviliza el actor, y ni tan siquiera sus contextos o posiciones sociales, puesto que la previsión de la acción futura de un actor "surgiría" de la combinación de una miríada de trazas pasadas que ha dejado en el mundo. El pasado del individuo, medido en tiempo real a través del análisis de sus diferentes trazas (sobre todo informáticas), permitiría anticipar su acción futura. No importan ya las razones o los motivos, puesto que es posible, haciendo la economía de ellas, prever las conductas. Obviamente, el aggiornamento de la antigua tesis conductista es patente. Sin embargo, la novedad está en otra parte: en la pretensión de hacer posible este proyecto gracias a las inéditas posibilidades técnicas actuales.

Detrás de esta posibilidad factual, se desliza así la idea que ya no es más necesario escrutar la conciencia o encuadrar los cuerpos (la lógica de la confesión o de las disciplinas de las hablaba Foucault, 1975 y 1976) para gobernar a los hombres. Las correlaciones exhaustivas y en tiempo real del tracking serían suficientes para hacer realidad el Panóptico de Bentham.

En la autoridad-relacional el consentimiento (voluntario o manipulado) es un factor decisivo; en la autoridad-factual del tracking la espinosa búsqueda del consentimiento desaparece detrás de la idea que es posible prever, gracias a esta técnica, las conductas futuras. La interioridad privada cede el paso a las superficies calculadas desde las trazas del Yo, lo que engendra una dialéctica particular entre las experiencias personales vividas y los datos exteriorizados calculados (Licoppe, 2013): a término, no es más necesario organizar el consentimiento puesto que las acciones se gobiernan gracias a la anticipación por tracking de conductas pasadas.

Si esta representación termina por imponerse, estaríamos delante de una de las más grandes transformaciones culturales de la representación del Yo en Occidente. Desde Agustin y Las confesiones, la subjetividad estuvo asociada a la exploración de un fuero íntimo, merced a una introspección, que asoció la interioridad con la imagen de la profundidad. El Quantified Self rompe con 
esta tradición: la subjetividad se devela por sus trazas exteriorizadas, o sea que el Yo se asocia con la imagen de la superficie. Resultado: los resortes de la acción no deben más descubrirse a través del estudio de la conciencia (o de su orientación por la inculcación ideológica o la legitimidad), sino deben establecerse, gracias a la mera multiplicación de correlaciones, desde las trazas exteriorizadas pasadas.

Lo que hicimos, en verdad, las trazas de lo que hicimos, permitiría prever lo que haremos. Nuestras compras anteriores permiten, por ejemplo, a los algoritmos prever nuestras compras futuras (incluso comparándolas con las de otros consumidores con gustos análogos); nuestras sucesivas localizaciones en el espacio, gracias a los GPS, permiten prever nuestras circulaciones futuras; nuestro historial bancario o nuestra presencia en cualquiera otra base de datos permitiría prever nuestras conductas futuras... Y así en más. En este universo, la autoridad, como consentimiento crítico, pierde todo valor (y función) en beneficio de la mera auscultación fáctica de las trazas del pasado como vía para anticipar el futuro.

El control de las trazas en internet concierne, así, el trabajo, la seguridad, la diversión, la salud, la propaganda política ${ }^{5} .$. Estas trazas son reputadas permitir conocer, a ciencia cierta, o sea, a través del examen de las conductas reales pasadas de un individuo, el futuro de sus conductas. Para ello se construyen, con la ayuda de correlaciones más o menos sorprendentes, perfiles de individuos en términos de longevidad de vida, salud, orientación política, pero también, perfiles de deudores. Un análisis de trazas que se apoya tanto en la información consentida y transmitida por un usuario, en el tratamiento secundario de información recuperada y analizada por terceros, o incluso de información involuntariamente capturada o desviada sin ningún consentimiento por el usuario. Lo importante es que estos datos permiten diseñar perfiles que no son predefinidos (o sea, que no remiten a identidades sociales usuales) sino que producen por correlación de una gran masa de datos perfiles inéditos y extraños por simple recombinación de factores. Es el principio mismo de la exploración de los Big Data: el tratamiento automático de datos revela correlaciones imprevistas entre los hechos. Resultado: el conocimiento altamente singularizado y predictivo, y la gestión proactiva, de las acciones futuras de un individuo, se apoya sobre la suposición de la existencia de una

5 Piénsese, incluso, en la manera como se controlará mañana, tal vez, la buena “conciencia” ecológica de los ciudadanos, gracias a una pulga RIFD que permitirá seguir desde su construcción hasta su desecho, el itinerario de los objetos (y por ende, controlar por tracking de conductas si el usuario ha o no separado los productos al echarlos). 
fuerte similitud de conductas entre los actores que poseen un mismo "perfil" (Bruno, 2014; Bigo, 2014). La lógica del tracking promueve un gobierno de los hombres a través del futuro anticipado.

En el universo del tracking generalizado, al menos como ilusión, todas las conductas dejan trazas y por ende todas las acciones pueden ser objeto de vigilancia. Las conversaciones telefónicas como los e-mails, los desplazamientos físicos como el uso de las tarjetas bancarias, las prácticas de consumo (tickets) como los datos presentes en las redes sociales o en internet. Sin lugar a dudas, y sin que sea necesario caer en visiones inútilmente extremas, las capacidades de vigilancia y de control de los actores se han incrementado masivamente en las últimas décadas. Cierto, las mismas técnicas permiten la expresión de movimientos de protesta o de denuncia (de Wikileaks al caso Snowden), pero tal vez lo importante, para el tema de la autoridad, se encuentre en otro registro. Más allá de los graves riesgos que estas denuncias han hecho visualizar a nivel de las libertades ciudadanas, al hacer públicas las capacidades exponenciales de vigilancia, estas denuncias contribuyen, sin duda involuntariamente, a asentar la idea de una sociedad gobernada por el tracking permanente y sin desmayo de todas las conductas. Lo que refuerza, paradójicamente, incluso sin duda más allá de las capacidades técnicas y organizacionales actualmente disponibles, el imaginario de una sociedad de control todopoderosa.

Se trata de un cambio importante en la gestión del consentimiento de los subordinados. Era, por ejemplo, a través de la legitimación de una cultura arbitraria de clase que la escuela participaba en reproducción social. Una función que cumplía tanto mejor mientras más la disimulaba por diferentes procesos de desconocimiento que aseguraban la correspondencia funcional entre la sociedad y el sistema educativo. La supuesta neutralidad de la escuela era descrita así como un engaño necesario para el funcionamiento del sistema (Bourdieu y Passeron, 1970). Muy distinta es la situación cuando el hecho de hacer visibles los controles se convierte en sí mismo en un mecanismo de control ${ }^{6}$. Los individuos tienen entonces menos el sentimiento de estar enfrentados a procesos opacos u ocultos, que el de encontrarse desprovistos de recursos para hacer frente a un sistema de control tentacular que, apoyándose

6 Es una consecuencia de un control que busca ejercerse menos por la vía del consentimiento, que por coerciones cada vez más experimentadas y presentadas como coerciones inevitables. Por supuesto, es a todas luces falso afirmar que no se impone ya ningún sistema de dominación cultural. En muchos ámbitos, la vida social sigue marcada por principios sociales o culturales que operan como un verdadero molde al cual los individuos deben adaptarse. Pero en lo sucesivo hay que agregar a los procesos de imposición ideológica los efectos inducidos por esta nueva modalidad de toma en cuenta de las coerciones. 
en lo que hicieron, gobierna por anticipación lo que harán. La obra de ciencia ficción de Philip K. Dick, Minority Report, resume a cabalidad esta filosofía y sin duda esta distopía: con el fin de evitar futuras acciones criminales, y gracias al tracking de conductas pasadas, se encierra o castiga por prevención, y antes de todo paso al acto efectivo, a ciertos individuos ${ }^{7}$.

En resumen, el tracking, gracias al análisis exhaustivo de las conductas pasadas, permitiría un gobierno tan eficaz de los hombres que la vieja autoridadrelacional, en tanto que búsqueda política de un consentimiento consentido entre individuos, se volvería un recurso innecesario.

\section{Dispositivos}

Para comprender la novedad de los dispositivos, tanto en la vida social en general, como a propósito de la autoridad, es útil regresar sobre la noción de reificación. En la acepción de Lukács, la noción destaca la tendencia fundamental del capitalismo a tratar a los individuos como cosas y, sobre todo, a abordar las relaciones entre individuos como meras relaciones entre cosas. La afirmación de Marx según la cual el problema no son los hombres sino el sistema, puesto que cualquiera que sean las cualidades morales personales de un patrón se encuentra obligado a comportarse como un patrón (a riesgo de ver quebrar su empresa), va, por supuesto, en esta dirección. La reificación designa, pues, un sistema total de coerción que oprime a todo el mundo, cierto que en el marco del marxismo en beneficio objetivo de una clase sobre otra, pero a través de un proceso que restringe a todos los individuos más allá de sus orientaciones morales. Lo fundamental es pues comprender que esta coerción al inscribirse en las cosas mismas (en latín -res), aparece como un mero resultado impersonal e inevitable del funcionamiento mismo del mundo. La coerción se presenta como un límite y un determinante fáctico de la acción (Courpasson, 2000: 24). No son las reglas heredadas del pasado o las reglas formales de la organización las que dictan la acción; ésta está directamente determinada por elementos exclusivamente factuales. Sigilosamente, la cuestión de la autoridad deja de ser un asunto normativo (lo permitido o lo prohibido), para convertirse en un asunto fáctico (lo posible y lo imposible). El actor, individual o colectivo, está obligado a someterse a restricciones exógenas factuales frente

$7 \quad$ No es el objeto de este artículo pero este mismo principio, incluso desde otras bases, se encuentra en los proyectos de la guerra preventiva, la tolerancia cero o los despistajes precoces, entre otros. 
a las cuales experimenta un sentimiento de impotencia. No se puede ir contra los "hechos".

Es aquí que los dispositivos obtienen todo su interés. Entendamos los dispositivos como mecanismos que "hacen hacer" (Agamben, 2007) independientemente de toda adhesión consciente o voluntaria, como cuando los actores, por ejemplo, pliegan espontáneamente sus acciones a caminos técnicamente preestablecidos. Un proceso prolongado por lo demás por la expansión de tecnologías inteligentes ("smart") en muchas actividades sociales. No está de más dar una lista de ejemplos. Piénsese en el dispositivo de la moneda presente en muchos carritos de compras en los supermercados: para poder usar el carrito el usuario tiene que colocar una pieza, y si quiere recuperar su pieza tiene que traerlo de vuelta -su conducta está gobernada, independientemente de lo que piense del dispositivo, por la coerción práctica del mismo. $\mathrm{O}$ en la manera en que los urbanistas reducen la velocidad de los automovilistas al ingreso de una aglomeración: en vez de convocar su buena conciencia ciudadana, prefieren construir pequeños bloques de cemento con un árbol al centro - un dispositivo que de manera refleja e infra-consciente reduce la velocidad. $\mathrm{O}$ en las plataformas informáticas en donde cada vez es más frecuente la necesidad de llenar ciertas casillas sin las cuales el programa "no funciona", o bien, como en tantos otros casos, en donde lo que quiere hacer ("introducir" o "bajar") no se puede hacer a causa de limites facticos presentes a nivel del diseño del dispositivo. $\mathrm{O}$ en la manera cómo es posible técnicamente, pero aun no aceptado por los usuarios, la incorporación del test de alcoholemia en los mismos automóviles: pasado el umbral permitido por la ley, el auto simplemente no arrancaría. Inútil alargar la lista, lo importante es comprender lo que todos ellos implican en términos de autoridad: el gobierno de los hombres se vuelve aquí también un gobierno por las cosas que se realiza en el tiempo presente, gracias a coerciones fácticas, que operan -en muchoindependientemente de toda conciencia o voluntad.

El gobierno de los hombres no sólo se apoya sobre instrumentos materiales (Lascoumes, Galès, 2004), sino que se transforma progresivamente en una mera gestión técnica. Aquí está sin duda la gran fuerza de los dispositivos. Ahí donde las reglas normativas exigen formas de coherencia, los dispositivos, gracias a su inscripción factual, pueden hacer la economía de estos esfuerzos. Los dispositivos prolongan así las disciplinas, pero a diferencia de muchas practicas disciplinarias en las que la presencia de los hombres es patente, en los dispositivos se asiste a una radical ocultación de su presencia y ejercicio. Por ejemplo, regresemos a esta ilustración, si se "obedece" a las 
reglas de funcionamiento de una plataforma informática ello no se explica por una adhesión normativa, sino por una necesidad meramente factual. Sin su "respeto", el aparato "simplemente" no funciona. En el mundo moderno, y bajo la lógica de los dispositivos cada vez más habría un desplazamiento de la autoridad-relacional fundada en normas a una autoridad-factual inscrita en el funcionamiento mismo del mundo. Los dispositivos se convierten en el mejor soporte de la autoridad. Notémoslo: para Weber, a pesar de la fuerza del proceso de racionalización, la autoridad racional-legal es aún de índole reflexiva y crítica; la autoridad-factual oblitera toda dimensión de este tipo ${ }^{8}$. Se obedece por razones utilitarias, funcionales, pragmáticas - en verdad por lo que se vive como coerciones fácticas.

Lo importante no es más influir en la "conciencia” de los individuos, sino centrarse en las maneras de orientar-determinar el comportamiento de los actores en situación, incidiendo en sus conductas a través de los dispositivos. El "plan" (el decurso) de las conductas se inscribe en la materialidad del mundo. Se trata a término del tránsito de una concepción fenomenológica de la acción hacia una concepción ecológica de la conducta. Para parafrasear a Ortega y Gasset, el individuo es él y sus objetos. El agenciamiento con el entorno es tal que éste se interpreta como una extensión de la personalidad, al punto que el individuo no puede concebirse independientemente de los objetos con los que interactúa y que determinan su acción. Los procesos cognitivos no se limitan a lo que una visión "internalista" supone sino que, en mucho, transitan por mecanismos externalizados. Las capacidades cognitivas humanas no se reducen a las de un espíritu individual separado del mundo, sino que se inscriben en una trama de dispositivos, a través de la cual los actores actúan tanto con sus manos y sus ojos como con sus cabezas (Ogien y Quéré, 2005: 13). La acción humana es inseparable de un sistema cognitivo (Conein y Jacopin, 1994). Cierto, muchos trabajos sobre la acción situada o la cognición distribuida otorgan un rol significativo a los individuos ${ }^{9}$, pero en muchos de estos trabajos también es visible la visión opuesta: una en la cual los dispositivos terminan construyendo un universo paralelo y cuasi independiente de los actores sociales. Las máquinas se observan entre ellas, piensan, interpretan, deciden, aprenden, se relacionan entre ellas y comunican de manera autónoma. En muchos de estos estudios, no

8 La noción de confianza de Giddens (1994), en los sistemas expertos da cuenta, de una manera sin duda problemática, de esta evolución, puesto que Giddens entrevé en ello una forma de consentimiento generalizado a los dispositivos técnicos.

9 El punto excede nuestro artículo, pero precisemos que en los estudios sobre la ecología de la acción las diferencias son notorias entre los estudios de psicología ecológica y los trabajos de la "cognición distribuida”, según el papel justamente que se otorgue a los objetos o a los sistemas cognitivos exteriorizados en la coordinación de las acciones. 
se trata pues solamente de tomar en cuenta el papel de los entornos técnicos, en aras de una concepción más equilibrada de las capacidades respectivas del agente humano y del entorno (lo que lleva a relativizar la atención exclusiva que se dio en el pasado a la capacidad intencional y voluntaria de los seres humanos) (Thévenot, 2000: 232). A término se trata de otra concepción de la acción y de su gobierno.

En lo que nos interesa, los dispositivos transforman el problema mismo de la autoridad. La espinosa cuestión de la desviación humana, tiende a ser controlada de otra manera. En efecto, no sólo en la coordinación de las acciones el papel dirimente le toca a los objetos, sino que subrepticiamente la crítica de la reducción de la vida social a la sola interacción entre humanos, abre en dirección de una concepción en la cual los objetos se convierten en los verdaderos "actores" de la sociedad. Solo a este precio, la sociología podría romper con su inveterado antropocentrismo. El gobierno de los hombres deja de ser un problema relacional y normativo, o sea de autoridad, para volverse un asunto de ingenieros -redes, asociaciones, instrumentos, por supuesto, dispositivos. Incluso las categorías normativas o administrativas son leídas desde su estricta funcionalidad: la acción "correcta" no se establece más en referencia a una norma (lo permitido o lo prohibido) sino en referencia a una exigencia fáctica (lo posible o lo imposible). Un proceso que permite la disociación entre la conciencia y las acciones: es así como, por ejemplo, durante el consumo un individuo puede sentirse particularmente libre puesto que se encuentra en tren de escoger entre diferentes productos, pero en ese mismo momento es objeto de un sinnúmero de controles y dispositivos que predeterminan su conducta independientemente de su conciencia (ubicación de los productos, envoltorios, publicidad...) (Bauman, 1988).

Por cierto, insistamos, no se trata de afirmar bajo ningún punto de vista que los estudios sobre la cognición distribuida, y aún más sobre la acción situada, niegan todo rol a las normas sociales. Sin embargo, detrás de la búsqueda de un equilibrio entre normas y dispositivos, autoridad-relacional y autoridad-factual, se impone muchas veces la tentación de eliminar los "actores" en beneficio de los objetos. Les prácticas no son más la aplicación de normas; a lo más se trata de "convenciones" que se apoyan y toman forma gracias a los objetos o dispositivos. Pocas tentativas han sido más explicitas a este respecto que el trabajo de Latour y su proyecto de romper con la vieja dicotomía entre la sociedad y el individuo, introduciendo -recuperando- lo que estas representaciones habían dejado de lado, a saber la "interobjetividad", el entorno de objetos sobre los que reposa la vida social. Para Latour (1994: 
598) es el "olvido de los artefactos (en el sentido de cosas) lo que ha creado este otro artefacto (en el sentido de ilusión): una sociedad que sería necesario estabilizar a través de lo social". En breve, la sociedad se mantiene gracias a una serie de asociaciones materiales entre "actantes" humanos y no humanos. Para Latour, esto exige dejar de pensar los objetos como puros intermediarios entre los humanos, y al contrario, y en una inversión de la célebre formula de Durkheim, tratar las cosas como hechos (y actores) sociales.

El punto de partida de esta visión puede ser aceptado sin dificultad: los intercambios conscientes entre individuos no son sino una parte de la vida social, y un buen número de nuestras interacciones se organiza a través de objetos y de sistemas independientes de nuestra conciencia. Pero de esta constatación, y en parte de esta negligencia por los objetos presentes en la sociología "clásica", se pasa a la afirmación que los objetos (los "actantes") deben ser considerados como verdaderos actores en la vida social (Latour, 1991 y 2006). La afirmación en su voluntad provocadora se presenta como una novedad radical, es una versión actualizada de la muy vieja tesis de las "estructuras" sin actores. Curiosamente la crítica de Latour hacia las estructuras se limita a rechazar la asociación de las "estructuras" con la acción de misteriosas "fuerzas", en aras de muy finas descripciones tangibles y factuales de la materialidad concreta del mundo, o sea, explicitar el trabajo de los objetos, por lo general técnicos, en el agenciamiento de la vida social. Pero detrás de este esfuerzo de descripción fáctica, la visión es profundamente tradicional: el viejo primado de las "estructuras" sin actores (propio a tantas visiones anti-humanistas, deterministas y estructuralistas) cambia de vocabulario, pero no de representación, con la teoría del actor-red en donde los objetos y los dispositivos se vuelven verdaderos "actores". En el fondo, la crítica al "antropocentrismo" de la sociología sólo sirve para actualizar una nueva versión del muy viejo anti-humanismo. Por lo demás, y aquí también en profunda continuidad con la sociología clásica, la pregunta por el orden social sigue siendo central en estos estudios: a lo más su explicación se desplaza desde los individuos hacia los objetos. En verdad, a una muy sutil articulación ontológica entre los individuos-y-las-cosas. De este modo, es fácil comprender por qué en el marco de esta filosofía de la vida social la autoridad-factual puede llegar incluso a remplazar lisa y llanamente a la autoridad-relacional.

Algo ha pasado, y grave, en el pensamiento social para que estas nuevas versiones de la muy vieja reificación del mundo gocen de un tal crédito. ¿Quién puede negar que la acción humana se apoye en "objetos" -instrumentos se decía ayer...- y que por ende incluirlos en la interpretación con el fin de eliminar esta zona de sombra es un avance metodológico y analítico? Pero ¿qué entonces de 
otros sistemas de relaciones sociales? ¿Estamos seguros que el crack bursátil se explica más por los dispositivos técnicos (o por la cocaína que consumen los traders...) que por las relaciones sociales propias al capitalismo financiero? ¿Por qué habría que privilegiar la articulación entre el individuo-y-sus-objetos en detrimento de otras relaciones sociales, históricas y estructurales a la hora de comprender y describir las acciones? Más allá del talento de Latour (1992), el proyecto Aramis (nombre de un medio de transporte colectivo técnicamente en "avance" sobre su época) fue abandonado no porque no se "lo amó suficiente", sino, mucho más banalmente (quiero decir “antropocéntricamente”...) porque la decisión no le compete nunca a los objetos per se, y a sus cualidades intrínsecas, sino a las relaciones sociales las cuales bañan los objetos y de las cuales son mediaciones. Si la cultura literaria de los defensores de esta visión del mundo fuera mayor, comprenderían que su utopía, indisociablemente política y científica, es la del ya muy viejo universo del nouveau roman. No el de un mundo de objetos-actores, sino de objetos en ausencia de actores. En verdad, y para ser más precisos, un mundo de actores limitados por otros actores a través del escudo de los objetos.

En este apartado nuestro objetivo no es empero llamar la atención sobre estos estudios sociológicos en particular, sino presentar la manera cómo la representación cultural del mundo de los dispositivos, y su papel en el gobierno de las conductas, se forja. Una en la cual, y detrás de la fascinación por los dispositivos, se vislumbra un evidente determinismo tecnológico y muchas veces incluso una sorprendente ingenuidad analítica -al final de cuentas, la concepción de los objetos-como-actores, y de los dispositivos como corazón del imperio de la autoridad-factual no son sino una versión anti-humanista y tecnófila a la moda de la muy vieja tesis del remplazo de la administración de los hombres por la administración de las cosas. Lo importante no es denunciar lo que esta sociología expresa, sino comprender los fenómenos sociales de los que estos estudios se invocan voluntariamente voceros.

\section{Ligaduras}

El tercer tipo de autoridad-factual toma la forma de una lógica de ligaduras. Para comprenderla, no está de más hacer referencia al estudio de Jon Elster (1998) sobre Ulises y las sirenas y la racionalidad imperfecta. Inspirándose de un célebre pasaje de la Odisea, Elster describe la racionalidad auto-restringida en analogía con una acción de Ulises, quien luego de haber colocado cera en los oídos de sus compañeros de viaje para que no escuchen los 
cantos de las Sirenas, se ata al mástil de su navío con el fin de escucharlas, pero no sin antes haberles dado la orden explicita que, pase lo que pase, y diga lo diga, nadie deberá acatar sus órdenes futuras. Para Elster, cuya lectura difiere sustancialmente de la propuesta unas décadas antes por Adorno y Horkheimer (1974), es con el fin de controlar las debilidades posibles de su voluntad que el actor decide subordinar sus acciones futuras a una decisión presente.

En su origen y en apariencia, las ligaduras no son pues "cosas", pero como lo veremos, y como lo connotan las ligaduras de Ulises, aquí también el gobierno por las cosas suplanta sigilosamente al gobierno por los hombres. En el mundo contemporáneo es sobre todo en el ámbito económico que esta lógica se expande. El futuro se encadena al presente: un procedimiento tanto más eficaz que las ligaduras -a la diferencia de lo que ocurre en el poema homérico- tienden a ser progresivamente olvidadas y "naturalizadas".

Partiremos aquí también de algunos ejemplos. A través de distintos mecanismos, ciertas decisiones económicas importantes tienden a someterse al diktat automático de la evaluación por indicadores (Martuccelli, 2010). De lo que se trata es de imponer decisiones imperativas bajo el manto de coerciones fácticas. Es así como, por ejemplo, ciertos fondos de inversión exigen tasas de rentabilidad anuales ex ante a las empresas, o sea imponen objetivos de beneficios - como ligaduras- independientemente de la coyunturas ${ }^{10}$. $\mathrm{O}$ de manera aún más imperativa, se inscriben en las constituciones principios económicos (bajo la forma de ciertos indicadores) transformándolos así en objetivos imperiosos. Las decisiones estratégicas se diluyen detrás de ligaduras fácticas.

Algunos tratados de la Unión Europea son una buena ilustración. Ya sea en nombre de la libre competencia o de la disciplina presupuestaria, los controles tienden a presentarse como necesidades fácticas. Desde el Tratado de Maastricht, por ejemplo, el déficit público no debe exceder el 3\% del PBI y a su vez la deuda pública no debe superar el $60 \%$ del PBI. De inmediato surge la pregunta, ¿por qué estas cifras? ¿Por qué 2 ó $4 \%$ para el déficit, y no 50 u $80 \%$ para la deuda? ${ }^{11}$ Por supuesto, se trata de ligaduras imperativas particulares puesto que muchos países de la zona euro se han "liberado" temporariamente de estos indicadores (empezando por Alemania y Francia en los años 2000, y tantos otros tras la crisis iniciada en 2007-2008); pero lo importante para

10 La tasa de rendimiento del capital -ROE, return on equity- rondó durante años alrededor del $15 \%$, una cifra significativamente más alta que el crecimiento del PIB.

11 Para una interpretación de la razón de estos umbrales, cfr. Cohen (1996: capítulo 7); cfr. también las reflexiones de Piketty (2013: 923-933). 
nuestro razonamiento es que estos indicadores se presentan como objetivos indispensables. El objetivo es volver automático el lazo entre la medida del resultado y la decisión política (Ogien, 2010).

O sea, es el umbral -la ligadura- lo importante. Cierto, es la política que los fija; y su carácter arbitrario es tan evidente que estos umbrales son objeto de legitima discusión entre los expertos (Reinhart y Rogoff, 2009; Ramaux, 2012); y para ciertos analistas incluso, detrás de esta ligadura del futuro se encontraría la voluntad de algunos grupos sociales de orientar las políticas económicas en su beneficio (Economistes Atterrés, 2011). Pero lo esencial para nuestro objetivo está en otra parte: en la voluntad de instaurar jurídicamente objetivos económicos inamovibles. Una posición inicialmente demandada por el propio Milton Friedman y su proyecto de inscribir en las constituciones la prohibición de todo recurso a lo que él denominaba la emisión inorgánica de la moneda.

En lo que concierne al problema de la autoridad, lo esencial reside en la voluntad de plegar las acciones futuras a una decisión presente; o sea, en sustraer del debate político futuro ciertos temas transformándolos en imperativos. Si en este proceso, los umbrales en sí mismos no significan gran cosa, la entronización de los indicadores como objetivo imperativo es una novedad importante. La importancia de este proceso es tal que la prensa europea no tardó en llamar a estos umbrales-obligaciones la "regla de oro".

Evitemos toda confusión. Las democracias modernas se apoyan y se sostienen gracias a la evicción de ciertos temas fuera del espacio de la deliberación política - piénsese en los Derechos Humanos cuya carácter es intangible cualquiera que sea la mayoría que acceda al gobierno. Sin embargo, en este caso se tratan de garantías colectivas recíprocas que los ciudadanos se otorgan entre sí merced a las instituciones; libertades de base cuyo cuestionamiento socavaría los fundamentos mismos del pacto político. ¿Es necesario decirlo? Nada semejante ocurre con la sacralización constitucional de los indicadores económicos.

En realidad, detrás y a través de la lógica de las ligaduras se vislumbra una de las grandes transformaciones de las últimas décadas: la gobernabilidad de la sociedad ya no opera más por, sino para el mercado. Un modo de intervención política que se caracteriza, curiosamente, por limitar el campo de intervención sobre la economía. Como en la racionalidad-ligada de Ulises, aquí también se construye una economía basada en ligaduras que, dictadas por la política, 
se presentan y se legitiman empero como siendo puramente económicas -fácticas- e independientes de la política. En un solo y mismo movimiento, la regla soberana es a la vez enunciada por la política y confiscada a la política. Ya no se trata más de gobernar lo menos posible (como en el liberalismo clásico), sino de sustraer el mayor número posible de decisiones económicas al juego político... a través de decisiones políticas ${ }^{12}$.

Si nos hemos centrado en este ejemplo es porque, más allá de su importancia, permite vislumbrar lo propio de la lógica de las ligaduras. A saber, la construcción y la naturalización de ciertos indicadores como principios imperativos sustraídos al debate público, que tienden progresivamente a actuar como diktats fácticos independientemente de todo consentimiento crítico, o mejor dicho, que tienden incluso a operar en ausencia de consentimiento por la "fuerza misma de las cosas". Un conjunto muy importante de indicadores económicos (piénsese en el "riesgo país", en las notas de las agencias de evaluación financieras...) tienden así a operar, "inmediata y naturalmente", como si fueran bornes fácticos. El valor de las transacciones en la bolsa, las tasas de cambio, la entrada o la salida de capitales tienden a presentarse como sanciones "objetivas" de las políticas de un país -haciendo así olvidar el carácter ampliamente político ${ }^{13}$, subjetivo e incluso mimético de estas conductas (Orléan, 2011).

Lo esencial, por supuesto, es la transformación que esto supone a nivel de la autoridad. El consentimiento critico no es más indispensable, puesto que las decisiones las "imponen" las coerciones fácticas. El contraste es así notorio entre las movilizaciones que suscitaron las intervenciones del FMI en los años 1980 y la situación actual, en donde, incluso cuando existen movilizaciones, la responsabilidad se diluye en un gran número de actores, difícilmente identificables, y en donde sobre todo, el abanico posible de las decisiones parece estar directamente dictadas por coerciones fácticas insuperables. Es así, por ejemplo, que cuando una crisis se desata una de las grandes preocupaciones de los gobiernos actuales es la de preservar los equilibrios macroeconómicos (fiscalidad, moneda, balanza de pagos...) con el fin de no aumentar el indicador de "riesgo país". Todo error político en el manejo de la economía sería, en

12 Desde fines de los años 1990, Thomas Friedman (1999: 87) se refería a estas ligaduras como una "camisola de fuerza en oro" (Golden Straitjacket). Para Friedman, este tipo de medidas, que él asociaba con la globalización, suponían una expansión de lo económico y un encogimiento de la política- en efecto, una vez vestida, la camisola de fuerza de oro, restringía el margen de acción de la política.

13 En enero de 2011, por ejemplo, en plena revolución de la primavera árabe en Tunes, la agencia Moody's degradó la nota del país de Baa2 a Baa3 (Bronstein, 2014: 44). 
efecto, "inmediata y objetivamente" sancionado por los mercados. Un proceso reforzado por la incitación, a través de los organismos internacionales, de la adopción generalizada de best practices a nivel mundial. Lo importante, para nuestro propósito actual, es comprender la "naturalización" coercitiva que esto supone del proceso de decisión, y tras él, una nueva modalidad de evaporación de la autoridad-relacional. En todos estos procesos, una misma lógica se afirma: la confusión entre los medios y los fines. Los indicadores que al principio, y en principio, solo sirven para medir una tendencia, se transforman en los objetivos imperativos que deben alcanzarse.

Por supuesto, la lógica de las ligaduras presenta algunos puntos comunes con el proceso de racionalización propiamente moderno. Pero la radicalización del proceso, vía los indicadores, da paso a un cambio cualitativo: la autoridad -y el consentimiento crítico que ésta supone- se diluye detrás de la mera aplicación a cualquier tipo de organización (empresa, hospital, escuela, tribunal, comisariado, incluso país...) de las mismas prácticas de gestión mundializada. Las best practices son, así, el estadio final de la one best way taylorista. Tras su generalización se impone la idea que los imperativos económicos pueden ignorar los contextos sociales. Williamson, el inventor del consenso de Washington, pudo así definir su acuerdo como el consenso de sabiduría adoptado por todos los economistas serios.

Por supuesto, en la medida en que todo indicador procede de una elección política, incluso por omisión, es posible argüir que la dimensión propiamente política reside en el momento de construcción de los indicadores (lo que invitaría a un conjunto de ejercicios críticos sobre, por ejemplo, los indicadores económicos que deben privilegiarse -Gadrey, Jany-Catrice, 2005; Salama, 2006). Sin embargo, con el tiempo, los indicadores tienden a confundirse con imperativos fácticos y solo fácticos. Con lo cual, como es el caso por ejemplo de los indicadores de déficit público y de endeudamiento en la Unión Europea, el momento político se escamotea detrás de una decisión que aparece como meramente técnica y dictada imperativamente por la "fuerza de las cosas". Es bajo esta modalidad que las ligaduras transforman el gobierno de los hombres en el gobierno por las cosas.

Nada resume mejor este proceso, en su voluntarismo fáctico, que el famoso acrónimo TINA- There is no alternative ${ }^{14}$. A través del recurso a esta fórmula, y más allá de sus evidentes aspectos políticos o ideológicos, e incluso técnicos o científicos, lo importante es la transformación de la autoridad-

14 Para un balance crítico de treinta años de uso de este acrónimo, cfr. Rothé y Mordillat (2011). 
relacional en una mera autoridad-factual: la acción está determinada por los imperativos ineluctables de la realidad. Y es para enfrentarlos, pero adaptándose a ellos, que las sociedades deben -como Ulises- ligar sus acciones futuras para no desviar de rumbo. La realidad, el orden factual presente, dicta el curso de la acción futura desde el horizonte de lo posible y de lo imposible. Reconozcamos que el éxito progresivo de la lógica de las ligaduras es asombroso. Pocas cosas son tan contundentes en el mundo de hoy que el recurso "ineluctable" a ciertas políticas que serían impuestas por el orden factual del mundo.

\section{Renovar la crítica}

Los tres mecanismos que hemos analizado aúnan a la vez prácticas efectivas y representaciones sociales. En cada caso, y por vías distintas, de lo que se trata es de sustraer el gobierno de los hombres a los cuestionamientos -inevitables- suscitados por la autoridad-relacional. A través de diferentes gestiones de los tiempos de la acción, el tracking (el gobierno desde las trazas pasadas), los dispositivos (el gobierno desde las acciones técnicas presentes) y las ligaduras (a través del encadenamiento del futuro) tienden a imponer el reino de la autoridad-factual.

El imaginario de la autoridad-factual está hoy lo suficientemente expandido como para que sea incluso posible diseñar tendencialmente una nueva geografía del ejercicio de la autoridad. Es en efecto posible afirmar que son los ámbitos "todavía" bajo la fuerte impronta de la autoridad-relacional - en mucho, la familia y la escuela, la civilidad urbana y en parte el trabajo- en donde el ejercicio de la autoridad aparece como más problemático; mientras que, ahí donde reinaría la autoridad-factual, la subordinación-coordinación de los hombres se efectuaría más fácilmente (seguridad, economía, marketing, disciplina...). Nada de extraño por ende que existan propuestas para expandir en los primeros ámbitos (familia, escuela, trabajo...) las ventajas de la autoridadfactual: generalización de cámaras, control de adulterios en línea o por las redes sociales, seguimiento de los adolescentes por GPS, vigilancia constante de los tele-trabajadores merced a la grabación de todas las llamadas o control de la velocidad de gestión de los dossiers gracias a mecanismos informáticos, gestión del ruido de los adolescentes en lugares públicos gracias a dispositivos 
sonoros que (inaudibles para los adultos) los haría desertar ciertos lugares, etc. En breve, el futuro pertenecería a la autoridad-factual.

Sin embargo, esta tendencia esconde peligros significativos. El gobierno de la ley -a través de la impersonalidad- y no de los hombres -o sea de su arbitrario- es una conquista de la civilización. Pero la impersonalidad de la ley -los célebres ojos vendados de la justicia- no debe confundirse con la autoridad-factual. En el primer caso, de lo que se trata es, por dificil que sea la ecuación, e incluso su imposibilidad, transformar el poder discrecional de unos hombres sobre otros en una autoridad-relacional críticamente consentida. En el segundo caso, por el contrario, lo que se trata es de sustraer la discusión sobre el poder de unos sobre otros en beneficio de una funcionalidad impuesta por las cosas ${ }^{15}$.

Esta sustracción parece, a primera vista, un blanco evidente y fácil de la crítica. Ya sea que se trate del tracking, de los dispositivos o de las ligaduras -y el orden factual al cual someten la triple temporalidad humana: pasado, presente y futuro- en cada caso bastaría con abrir la "caja negra" para asegurar el trabajo crítico y emancipador. En este sentido, es verdad que la crítica a la autoridadfactual no presenta en el fondo ningún nuevo desafío (al final de cuentas las sociedades han sabido resistir al determinismo político y económico, sabrán mañana resistir al determinismo de los artefactos). Sin embargo, las cosas, tratándose de hechos presentados como cosas, no son tan simples.

En primer lugar porque los actores mismos expresan anhelos en dirección de la autoridad-factual y en contra de la autoridad-relacional. Para muchos en efecto el rodeo por las cosas atenúa el carácter inevitablemente polémico de la autoridad en una sociedad igualitaria; su cosificación vuelve su práctica menos denigrante y más neutra en apariencia. A lo cual aún es necesario añadir el hecho que la autoridad-factual, en su expansión contemporánea, parece encarnar, infinitamente mejor que la autoridad-relacional el imaginario de un poder simétrico propio al ideal de la igualdad jurídica. Escollos sin duda significativos, la principal dificultad es sin embargo de otra índole. Resistir a la autoridad-factual supone revindicar y alegar en favor de la autoridadrelacional. Aquí está el meollo de la cuestión y el verdadero desafío al trabajo crítico. Paradójicamente, para simetrizar el ejercicio de la autoridad, es preciso introducir -reintroducir- los individuos por doquier. Proceso sin duda paradójico a ojos de muchos a tal punto la modernidad asoció la emancipación

15 En este sentido puede comprenderse la autoridad-factual como otra tendencia contemporánea hacia la post-democracia (Crouch, 2003). 
con el fin de la subordinación personal; a tal punto, como lo hemos evocado, el ejercicio de la autoridad-relacional es el blanco de múltiples procesos de contestación o de cuestionamiento.

Repitámoslo: la autoridad-factual, más allá de sus bases técnicas, debe entenderse también como una respuesta al incremento de las competencias críticas de los individuos y al trabajo cotidiano de denuncia de las injusticias (Boltanski, 1990). En la medida en que estas competencias se afirman, es preciso -desde un punto de vista funcional- asegurar y reforzar sobre otras bases la reproducción del orden social. Es lo que permite de alcanzar, justamente, el desplazamiento del consentimiento hacia factores que se consideran exclusivamente fácticos. Cierto, no hay que dramatizar la situación actual: cotidianamente, los actores continúan "consintiendo", "funcionando" y "respetando" a la mayor parte de las "autoridades" establecidas; pero continúan por sobre todo, y por razones únicamente fácticas, sometiéndose a las reglas de funcionamiento de la vida social. Pero ahí donde la percepción crítica de las relaciones de subordinación interpersonales no cesa de acrecentarse (incluso cuando los actores se pliegan a ellas), por el contrario, la neutralización de la mirada crítica gracias a la pretendida objetividad intrínseca del mundo es una respuesta innegable (incluso cuando los actores expresan malhumores). Las primeras deben ser constantemente reforzadas, haciéndose por lo mismo cada vez más visibles y menos "naturales"; las segundas, por el contrario, basta con que se repitan con frecuencia para que se vuelvan invisibles y "naturales". El trabajo de zapa de la crítica ha logrado que, al menos intelectualmente, ninguna autoridad-relacional se acepte por sí misma y que esté por doquier sometida al riesgo de la discusión, ahí donde, por el contrario, la autoridad-factual se acepta con una gran automaticidad y como bajo estrictas consideraciones funcionales.

Sin embargo, en este punto, el pasado debe servir, sino como guía, por lo menos como objeto legítimo de reflexión. Y es apoyándose en él, que es posible afirmar que el gobierno por los hombres enmarcados por la impersonalidad de la ley aparece como una mejor opción que la sustracción del gobierno de los hombres por las automaticidades factuales. Más vale ser evaluado por una "persona" que por un conjunto de indicadores; más vale someterse a una evaluación "subjetiva" que a un ranking "objetivo" (benchmarking); más vale poder exigirles cuentas a un gobernante (accountability) que someterse al dictado fáctico e inimputable de los dispositivos; más vale combatir la imposible erradicación de la desviación normativa humana que creer en la predicción absoluta de las conductas por el tracking generalizado de las trazas 
exteriorizadas; más vale seguir entendiendo la vida social como un conjunto estructurado de relaciones sociales conflictivas que como un ensamblaje dispar de actantes; más vale estudiar el proyecto de poder presente en la Razón técnica que limitarse a analizar la calidad de las conexiones, las piezas y las articulaciones técnicas. En el fondo, y bien vistas las cosas, más valen las inevitables "mentiras" de la política de los hombres que las ilusorias "verdades" de la ingeniería por las cosas.

Pero para ello, para todo ello, es necesario reintroducir los individuos y los sistemas de relaciones sociales en todos los ámbitos de la vida social de donde tienden a ser excluidos justamente por los defensores y los profetas del nuevo orden factual. Sí, el ejercicio de la autoridad entre los hombres es un desafío cotidiano en el mundo contemporáneo. Uno de los precios a pagar por la adhesión al ideal de la igualdad jurídica y política entre los hombres. Frente a este ideal, toda forma de subordinación, por consentida y crítica que sea, no dejará nunca de ser un escándalo. Pero ello no es ninguna razón para escaparse de esta aporía en aras de los impases que se esconden detrás de la ilusión de la autoridad-factual. Con todos sus peligros, limitaciones e inevitables dominaciones, más vale fundar la autoridad en el gobierno de los hombres que en la administración por las cosas. 


\section{Referencias bibliográficas}

Adorno Theodor W., Horkheimer Max (1974), La dialectique de la Raison [1947], Gallimard, Paris.

Agamben Giorgio (2007), Qu'est-ce qu’un dispositif?, Payot et Rivages, Paris.

Bauman Zygmunt (1988), Freedom, University of Minnesota Press, Minneapolis.

Bigo Didier (2014), "Sécurité maximale et prévention ? La matrice du futur antérieur et ses grillees”, in Barbara Cassin (dir.), Derrière les grilles, Mille et une nuits, Paris.

Boltanski Luc (1990), L’amour et la justice comme compétences, Métailié, Paris.

Pierre Bourdieu, Jean-Claude Passeron (1970), La reproduction, Minuit, Paris.

Bronstein Serge (2014), "Les grilles de notation financière", in Barbara Cassin (dir.), Derrière les grilles, Mille et une nuits, Paris.

Bruno Fernanda (2014), « Grilles de nos traces sur internet », in Barbara Cassin (dir.), Derrière les grilles, Mille et une nuits, Paris.

Cohen Elie (1996), La tentation hexagonale, Fayard, Paris.

Conein Bernard, Jacopin Eric (1994), « Action située et cognition. Le savoir en place », Sociologie du travail, n4, pp.475-500.

Courpasson David (2000), L'action contrainte, P.U.F., Paris

Crouch Colin (2003), Postdemocracy, Gius.Laterza \& Figli, Roma.

Elster Jon (1998), Ulysses and the Sirens [1979], Cambridge Paper Back, Cambridge.

Foucault Michel (1975), Surveiller et punir, Gallimard, Paris.

Foucault Michel (1976), Histoire de la sexualité, t-1, Gallimard, Paris. 
Friedman Thomas (1999), The Lexus and the Olive Tree: Undestanding Globalization, Farrar, Strauss and Giroux, New York.

Gadenne Emmanuel (2012), Le guide pratique du 2uantified Self, FYP Editions, Paris.

Gadrey Jean, Jany-Catrice Florence (2005), Les nouveaux indicateurs de richesse, La Découverte, Paris.

Giddens Anthony (1994),Lesconséquencesdelamodernité[1989], L’Harmattan, Paris.

Habermas Jürgen (1987), Théorie de l'agir communicationnel [1981], Fayard, Paris, $\mathrm{t}-2$.

Lascoumes Pierre, Le Galès Patrick (dir.) (2004), Gouvernerparlesinstruments, Presses des Sciences Po, Paris.

Latour Bruno (1991), Nous n'avons jamais été modernes, La Découverte, Paris.

Latour Bruno (1992), Aramis ou l'amour des techniques, La Découverte, Paris.

Latour Bruno (1994), « Une sociologie sans objet ? Remarques sur l'interobjectivité », Sociologie du travail, n4, pp.587-607.

Latour Bruno (2006), Changer de société, refaire de la sociologie, La Découverte, Paris.

Les économistes Atterrés (2011), «Réforme du Traité : une Europe plus solidaire... avec les marchés ? », www.atterrés.org.2011.

Licoppe Christian (2013), « Formes de la présence et circulation de l'expérience. De Jean-Jacques Rousseau au 'Quantified Self' », Réseaux, novembre-décembre, pp.23-55.

Mayern Schönberger Viktor, Cukier Kenneth, Big Data, Boston-New York, Houghton Mifflin Harcourt (2013).

Martuccelli Danilo (2001), Dominations ordinaires, Paris, Balland.

Martuccelli Danilo (2007), Cambio de rumbo, Santiago, LOM Ediciones. 
Martuccelli Danilo (2010), "Critique de la philosophie de l'évaluation", Cahiers internationaux de sociologie, CXXVIII-CXXIX, pp.27-52.

Marx Karl (1977), Le capital [1867], Editions sociales, Paris, t-1.

Ogien Albert (2010), « La valeur sociale du chiffre. La qualification de l'action publique entre performance et démocratie », Revue française de socioéconomie, ${ }^{\circ} 5$.

Ogien Albert, Quéré Louis (2005), Le vocabulaire de la sociologie de l'action, Ellipses, Paris.

Orléan André (2011), L'empire de la valeur, Seuil, Paris.

Salama Pierre (2006), Le défi des inégalités, La Découverte, Paris.

Piketty Thomas (2013), Le capital au XXI siècle, Seuil, Paris.

Ramaux Christophe (2012), L'État social, Mille et une nuits, Paris.

Reinhart Carmen M., Rogoff Kenneth S. (2009), This Time is Different. Eight Centuries of Financial Folly, Princeton University Press, Princeton.

Rothé Bertrand, Mordillat Gérard (2011), Il n’y a pas d'alternative, Seuil, Paris.

Thévenot Laurent (2000), « L'action comme engagement », in L'analyse de la singularité de l'action, P.U.F., Paris.

Weber Max (1971), Economie et société [1922], Plon, Paris. 